\title{
The effect of photon illumination in rapid thermal processing on the characteristics of MOS structures with ultra-thin oxides examined by substrate injection
}

\author{
Chia-Hong Huang ${ }^{\mathrm{a}, *}$, Jenn-Gwo Hwu ${ }^{\mathrm{b}}$ \\ ${ }^{a}$ Department of Electrical Engineering, Lunghwa University of Science and Technology, 300 Wan-shou Road, Sec. 1, Kueishan, \\ Taoyuan 33306, Taiwan, ROC \\ ${ }^{\mathrm{b}}$ Department of Electronics/Electrical Engineering, National United University, Miaoli 36003, Taiwan, ROC
}

Received 21 September 2004; received in revised form 18 April 2005; accepted 16 June 2005

Available online 6 September 2005

The review of this paper was arranged by Prof. Y. Arakawa

\begin{abstract}
The occurrence of the hysteresis-like breakdown (denoted by BD-H) and the minority carrier generation in MOS structures under substrate injection enhanced by the direct illumination treatment in a rapid thermal processing (RTP) are examined in this study. It is generally believed that the oxide breakdown under substrate injection is related to the oxide quality and the minority carrier generation contributed by bulk traps in Si substrate. For the breakdown behavior of MOS devices subjected by substrate injection, we find that the occurrence frequency of BD-H is more for the samples treated by the front-side heating (FH) processing than for those done by the back-side heating (BH) one in RTP. Besides, it is also observed that the gate saturation current is under the influence of the direct illumination processing. Moreover, from capacitance-voltage $(C-V)$ measurements with various frequencies, it indicates that the generation rate of minority carriers is higher for the samples treated by $\mathrm{FH}$ processing than for those done by $\mathrm{BH}$ one. These results show that not only localized oxide traps but also Si bulk traps are much easy to be created in the direct illumination processing. It is, therefore, clear that the characteristics of MOS structures with ultra-thin oxides are influenced by the direct illumination in RTP.
\end{abstract}

(C) 2005 Elsevier Ltd. All rights reserved.

PACS: $73.40 . \mathrm{Qv} ; 72.20 . \mathrm{Jv} ; 73.61 . \mathrm{Ng}$

Keywords: Illumination; Rapid thermal processing; Hysteresis-like breakdown; Minority carrier generation; Substrate injection; Bulk traps

\section{Introduction}

Owing to the demand of ULSI technology, rapid thermal processing (RTP) with the features of low-cost, low thermal budget, low-power consumption and high throughout plays a more and more important role in

\footnotetext{
* Corresponding author. Tel.: +8862 82093211x5529; fax: +8862 82099728.

E-mail address: chhuang@mail.lhu.edu.tw (C.-H. Huang).
}

the future. In an RTP with incoherent light as the source of energy, the generated microscopic defects in oxides are different from that in a conventional furnace. Moreover, the microscopic defects are much larger for RTP with back heating compared to that with front heating [1]. On the other hand, the effect of front- and back-side heating post-oxidation annealing, denoted by FPOA and BPOA, in RTP on ultra-thin gate oxides examined by gate injection has been reported [2]. Furthermore, an enhancement in soft breakdown occurrence has been 
also clearly observed for the samples treated by FPOA [2].

Various modes of oxide breakdown and minority carrier generation in MOS structures under substrate injection related to the illumination treatment during rapid thermal oxidation (RTO) and post-oxidation annealing (POA) processes have been investigated in this study. Generally, it is believed that the oxide breakdown under substrate injection is related to the oxide quality and the minority carrier generation contributed by bulk traps in $\mathrm{Si}$ substrate. It is found that the occurrence of hysteresislike breakdown [3], denoted by BD-H, is enhanced by the illumination treatment whether in RTO processing or in POA one. Besides, it is also observed that the gate saturation current is influenced by the illumination in RTP. Note that the gate saturation current is the gate current corresponding to the gate applied voltage of $5 \mathrm{~V}$. Additionally, from capacitance-voltage $(C-V)$ curves, it implies that the generation rate of minority carriers is higher for the samples treated by FPOA than for those done by BPOA. As stated above, it indicates that the illumination plays a significant role in RTP and affects the characteristics of MOS structures with ultra-thin oxides. Possible reasons for the observation are given later. It is, therefore, worthy to study the properties of the oxide traps and the Si bulk traps in MOS structures treated by the illumination treatment in RTP.

\section{Experimental}

The devices used in this study are metal-oxide-semiconductor (MOS) capacitors with an aluminum gate electrode fabricated on a $\left(\begin{array}{lll}1 & 0 & 0\end{array}\right)$ p-type Si substrate. The resistivity of the silicon substrate used in this study is $1-5 \Omega \mathrm{cm}$. Some gate oxides were grown by front-side or back-side heating rapid thermal oxidation, denoted by FRTO and BRTO, respectively, at $800-900{ }^{\circ} \mathrm{C}$ without POA. Moreover, the other gate oxides were grown by RTO at $800-900{ }^{\circ} \mathrm{C}$ for $35 \mathrm{~s}$, followed by front-side or back-side heating post-oxidation annealing in $\mathrm{N}_{2}$ ambience, denoted by FPOA and BPOA, respectively, at $960{ }^{\circ} \mathrm{C} 55 \mathrm{~s}$ [2]. The schematic diagram of front-side heating and back-side heating apparatus is shown in Fig. 1. Wafer temperature in an RTP system is measured with an IR pyrometer. Tungsten-halogen lamps which emit radiation with a peak at $1.0 \mu \mathrm{m}$, spectral distribution in the range of $0.5-1.0 \mu \mathrm{m}$, are used in the wafer heating system. After these treatments, the aluminum gate electrode was fabricated by thermal evaporation. The gate electrode area is $250 \times 250 \mu \mathrm{m}^{2}$. Aluminum was also used as the backside contact. The oxide thickness ranging from 1.8 to $3.9 \mathrm{~nm}$ was determined by ellipsometry. Current-voltage $(I-V)$ measurements with a ramp rate of $0.05 \mathrm{~V} / \mathrm{s}$ were carried out by using HP 4140B pA meter/dc voltage source. Before measuring
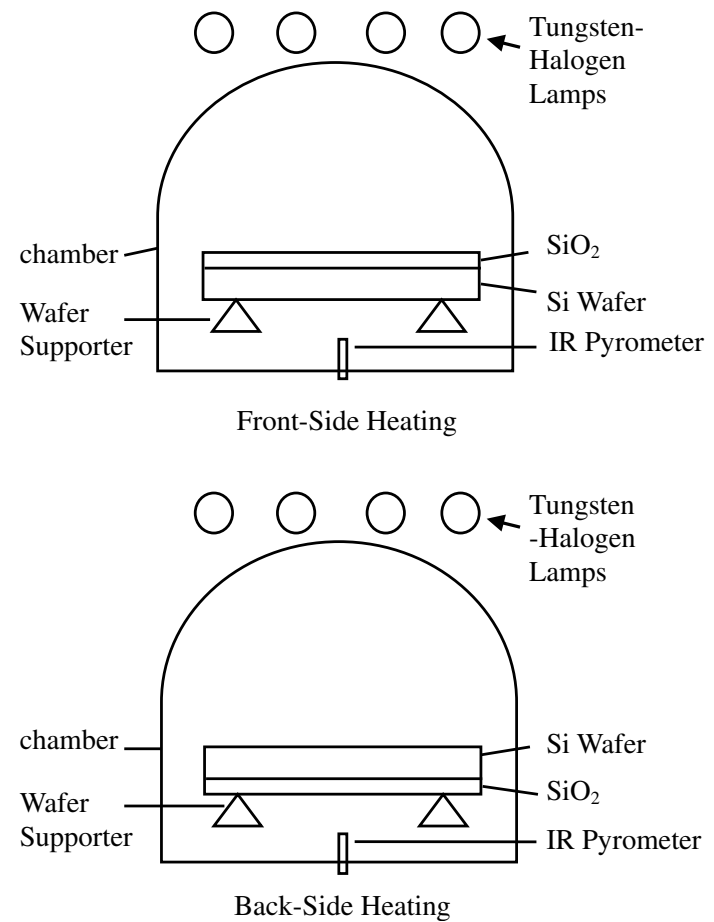

Fig. 1. The schematic diagram of front-side heating and back-side heating apparatus.

the $I-V$ characteristics, the gate was shorted to substrate for a few minutes to avoid transient effects $[4,5]$. Furthermore, $C-V$ curves were measured by using HP 4284A precision LCR meter. At least 30 devices were investigated for each process.

\section{Results and discussion}

Because of carrier tunneling and the limitation of minority carrier supply, it is observed that there are three different modes in $I-V$ characteristics of MOS capacitors under substrate injection [3]. They are resistor-like breakdown, hysteresis-like breakdown, and saturation without breakdown, denoted by BD-R, BD-H and NBD, respectively, as shown in Fig. 2 [3]. The occurrence frequencies of three modes are dependent on oxide thickness and are strongly related to the minority carrier generation near $\mathrm{Si}$ surface and also the oxide properties [3]. Fig. 3 shows the occurrence frequencies of three breakdown modes in 1.7, 2.1, and $3 \mathrm{~nm}$ oxide thickness for the samples treated by FRTO and BRTO. We find that the occurrence frequencies of the BD-H mode are more for the samples treated by FRTO processing than for those done by BRTO one. Besides, it is also obtained that the occurrence frequencies of the BD-R mode increase while the ones of the NBD mode decrease with oxide thickness.

For the samples treated by FPOA and BPOA, three breakdown mode relationships in 2,3 , and $3.9 \mathrm{~nm}$ oxide 

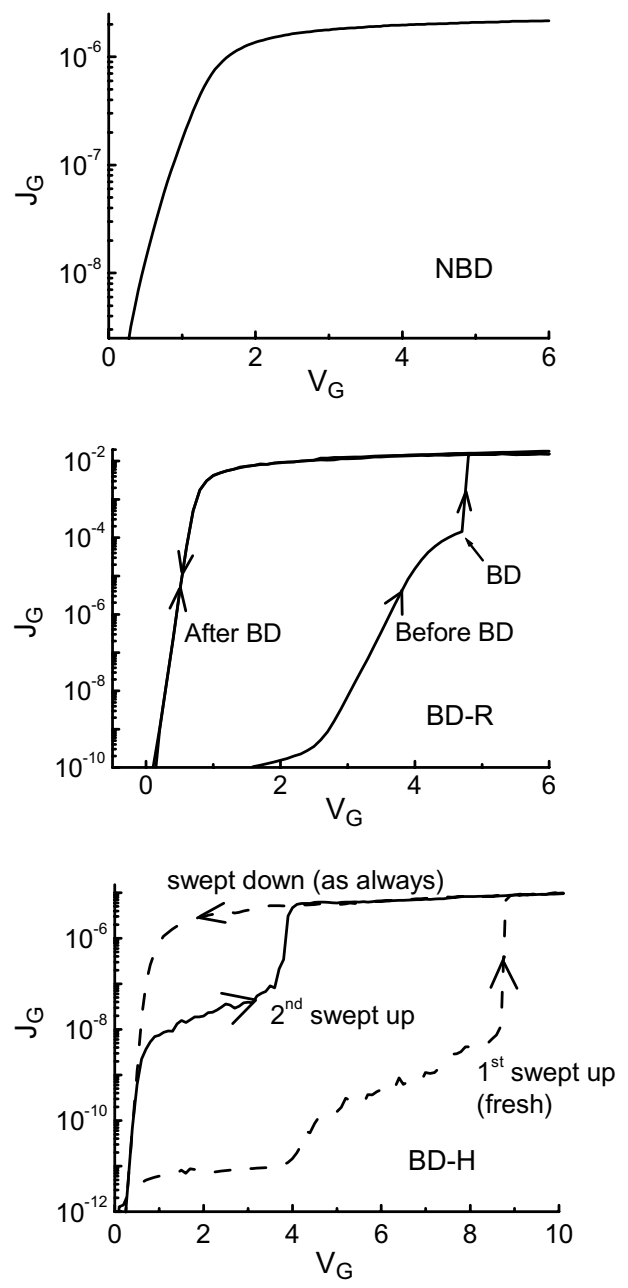

Fig. 2. The $I-V$ characteristics of the NBD, BD-H, and BD-R modes in MOS structures under substrate injection.

thickness are also illustrated in Fig. 4. The results are similar to those of the samples treated by FRTO and BRTO as mentioned above. Hence, it is worthy of noting that whether in RTO processing or in POA one, the occurrence frequencies of $\mathrm{BD}-\mathrm{H}$ are more for the samples treated by $\mathrm{FH}$ processing than for those done by $\mathrm{BH}$ one. It is because during the illumination treatment in RTP the distribution of oxide traps is influenced and then localized [2] so that it causes easily the occurrence of BD-H under substrate injection. The BD-H occurrence in MOS capacitors is, therefore, enhanced by the direct illumination treatment. On the other hand, it is generally believed that the oxide breakdown under substrate injection is related to the oxide quality and the minority carrier generation contributed by bulk traps in $\mathrm{Si}$ substrate. Consequently, the other reason for the above observation may be due to the difference in the supply of minority carriers generated by $\mathrm{Si}$ bulk traps between the samples treated by $\mathrm{FH}$ processing and those done by $\mathrm{BH}$ one. The effect of the illumination on the
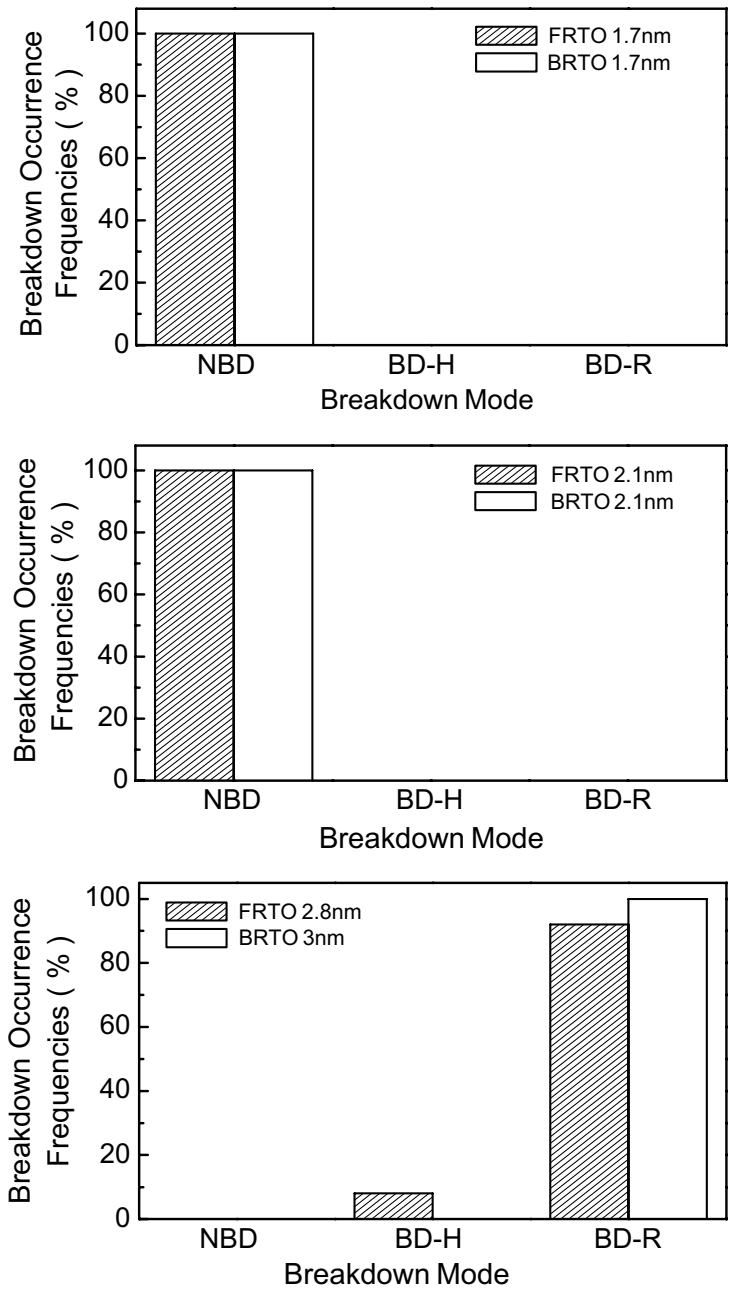

Fig. 3. The occurrence frequencies of three breakdown modes in 1.7, 2.1, and 2.8 (3) nm oxide thickness for the samples treated by FRTO and BRTO.

minority carrier generation of MOS devices is presented later.

As oxide thickness is less than about $2 \mathrm{~nm}$, oxide breakdown rarely occurs under substrate injection. The gate saturation current density versus oxide thickness for the samples treated by FRTO and BRTO is shown in Fig. 5. The inset depicts the typical $I-V$ characteristics of MOS structures treated by FRTO and BRTO with $2.1 \mathrm{~nm}$ oxide thickness. As a bias regime is in which the carrier tunneling is dominant, the gate current is almost the same for the samples treated by FRTO and BRTO. However, while a bias regime is in which the minority carrier generation is dominant, the gate current is dependent on the minority carrier generation. Additionally, the gate saturation current density versus oxide thickness for the samples treated by FPOA and BPOA is also shown in Fig. 6. The inset also depicts the typical $I-V$ characteristics of MOS structures treated by FPOA and BPOA with $2.0 \mathrm{~nm}$ oxide thickness. For the NBD mode, therefore, it is found that the gate 

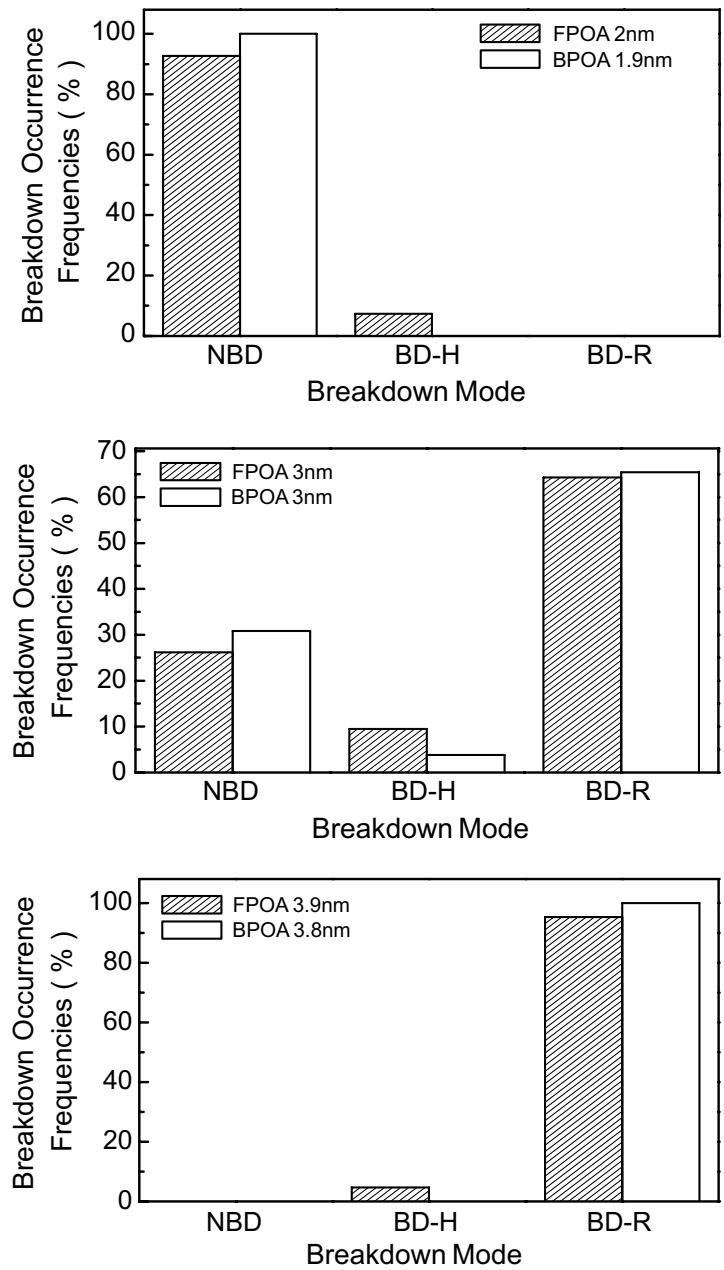

Fig. 4. The occurrence frequencies of three breakdown modes in 2 (1.9), 3, and 3.9 (3.8) nm oxide thickness for the samples treated by FPOA and BPOA.

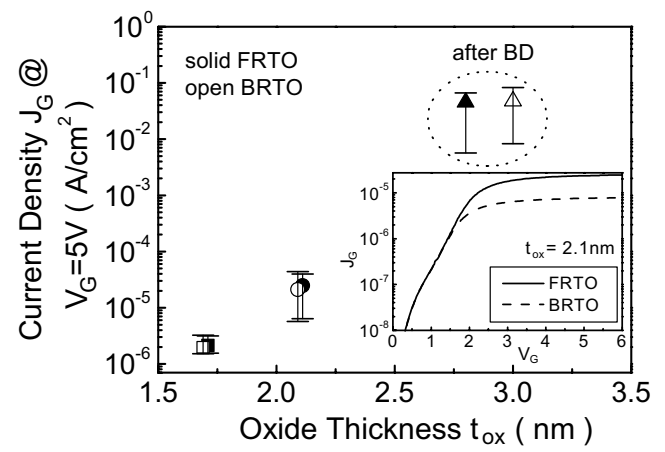

Fig. 5. The gate saturation current density versus oxide thickness for the samples treated by FRTO and BRTO.

saturation current is influenced by the illumination treatment whether in RTO processing or in POA one. It is noted that the gate saturation current is dependent on interface trap density and minority carrier lifetime [6]. These indicate that Si bulk traps in the substrate and interface traps at the $\mathrm{SiO}_{2} / \mathrm{Si}$ interface are also

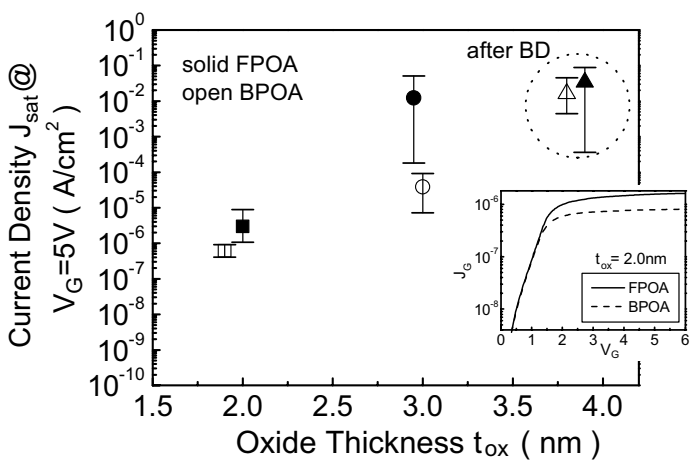

Fig. 6. The gate saturation current density versus oxide thickness for the samples treated by FPOA and BPOA.

under the influence of the illumination treatment. From previous results, therefore, it is obtained that not only oxide traps but also Si bulk traps near the interface are indeed influenced by the direct illumination treatment in RTP.

In the $C-V$ curves, generally, when the applied signal frequency is so low that the generation-recombination rate of minority carriers can keep up with the small-signal AC variation leading to charge exchange with the inversion layer. At higher frequencies, however, minority carriers cannot respond to the small-signal AC change. From this point of view, the generation-recombination rate of minority carriers can be examined by $C-V$ curves with various frequencies. $C-V$ characteristics are shown under various frequencies from 20 to $1 \mathrm{MHz}$ for MOS devices with $1.9 \mathrm{~nm}$ oxide thickness
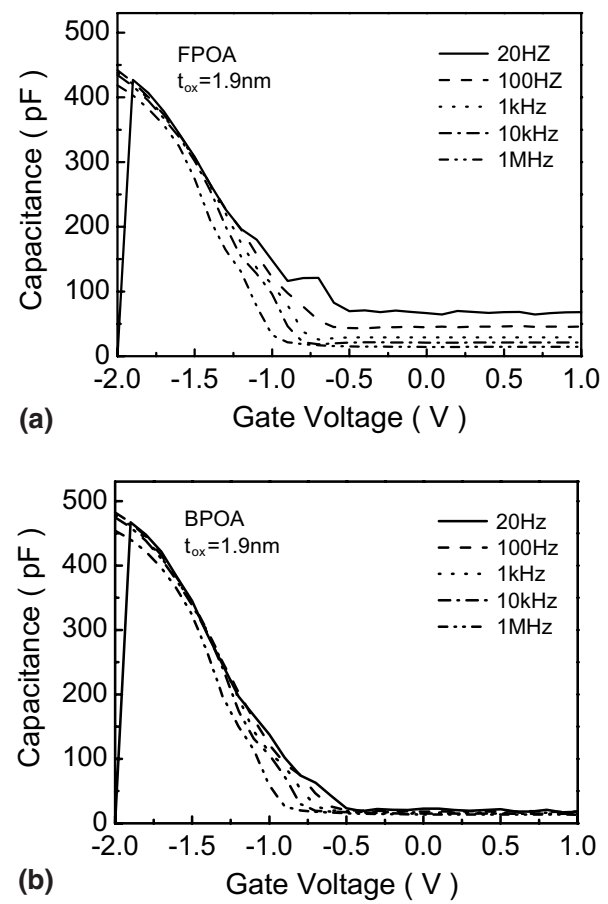

Fig. 7. Capacitance-voltage characteristics of MOS structures with $1.9 \mathrm{~nm}$ oxide thickness treated by (a) FPOA and (b) BPOA. 

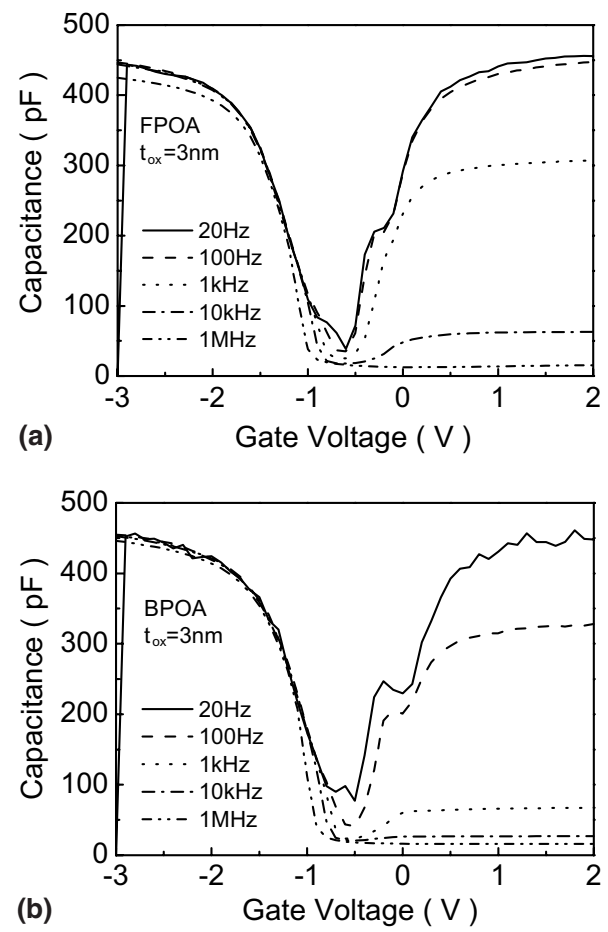

Fig. 8. Capacitance-voltage characteristics of MOS structures with $3 \mathrm{~nm}$ oxide thickness treated by (a) FPOA and (b) BPOA.

treated by FPOA and BPOA in Fig. 7(a) and (b), respectively. It is observed that the capacitance is larger for the samples treated by FPOA than for those done by BPOA in depletion and inversion region at lower frequencies $(20-1 \mathrm{kHz})$. The result implies that the amount of minority carriers is larger for the samples treated by FPOA than for those done by BPOA in the $C-V$ measurements. That is, the generation rate of minority carriers is higher for the samples treated by FPOA than for those done by BPOA. Similarly, for oxide thickness of $3 \mathrm{~nm}$, the above phenomena are also seen, as shown in Fig. 8 .

From the above discussion, it is obtained that the occurrence of $\mathrm{BD}-\mathrm{H}$ is enhanced by localized oxide traps caused by the illumination treatment and that the minority carrier generation is under the influence of the illumination processing. It is, therefore, proposed that Si bulk traps and oxide traps enhanced by the direct illumination during RTO/POA cause local current paths generated by localized weak spots through ultra-thin oxides, so the occurrence of BD-H is induced easily. Thus, the characteristics of MOS structures are indeed under the influence of the direct illumination in RTP.

\section{Conclusion}

The characteristics of the oxide breakdown and the minority carrier generation in MOS structures treated by the illumination processing under substrate injection have been investigated in this study. It is found that the occurrence of $\mathrm{BD}-\mathrm{H}$ is enhanced by the illumination treatment whether in RTO processing or in POA one. It indicates that the distribution of intrinsic oxide traps is influenced and then localized during the illumination processing in RTP. Meanwhile, it is also observed that the gate saturation current limited by the minority carrier generation is influenced by the direct illumination. From $C-V$ curves with various frequencies, moreover, it implies that the generation rate of minority carriers is higher for MOS structures treated by FPOA than for those done by BPOA. As a consequence, it is quite obvious that in addition to the influence on the oxide traps, the illumination also has a significant influence on the Si bulk traps. From the above description, it shows that photon energy plays a significant role in RTP and affects the properties of thin oxides and Si bulk in MOS structures.

\section{Acknowledgement}

The authors wish to thank the National Science Council of Republic of China for supporting this work under contract no. NSC 93-2215-E-262-001.

\section{References}

[1] Singh R, Nimmagadda SV, Parihar V, Chen Y, Poole KF. Role of rapid photothermal processing in process integration. IEEE Trans Electron Dev 1998;45(3):643-54.

[2] Huang C-H, Hwu J-G. Enhancement in soft breakdown occurrence on ultra-thin gate oxides caused by photon effect in rapid thermal post oxidation annealing. Solid-State Electron 2000;44(8): 1405-10.

[3] Huang C-H, Hwu J-G. Breakdown characteristics of ultra-thin gate oxide $(<4 \mathrm{~nm})$ in MOS structure subjected to substrate injection. J Vac Sci Technol 2001;B19(5):1894-7.

[4] Moazzami R, Hu C. Stress-induced current in thin silicon dioxide films. IEEE IEDM Tech Dig 1992:139-42.

[5] Riccó B, Gozzi G, Lanzoni M. Modeling and simulation of stressinduced leakage current in ultrathin $\mathrm{SiO}_{2}$ films. IEEE Trans Electron Dev 1998;45(7):1554-60.

[6] Dighish MY, Ho FD. A comprehensive analytical model for metalinsulator-semiconductor (MIS) devices. IEEE Trans Electron Dev 1992;39(12):2771-80. 